See discussions, stats, and author profiles for this publication at: https://www.researchgate.net/publication/332496574

\title{
Multi-lead ECG signal analysis for myocardial infarction detection and localization through the mapping of Grassmannian and Euclidean features into a common Hilbert space
}

Article in Biomedical Signal Processing and Control · April 2019

DOI: $10.1016 /$ j.bspc.2019.04.003

4 authors:

Panagiotis Barmpoutis

35 PUBLICATIONS 198 CITATIONS

SEE PROFILE

Anestis Apostolidis

Aristotle University of Thessaloniki

1 PUBLICATION 1 CITATION

SEE PROFILE

Some of the authors of this publication are also working on these related projects:

ProsocialLearn View project

Automatic seismic phase picking View project

READS

86

Kosmas Dimitropoulos

The Centre for Research and Technology, Hellas 114 PUBLICATIONS 902 CITATIONS

SEE PROFILE

Grammalidis Nikos

The Centre for Research and Technology, Hellas

158 PUBLICATIONS 1,549 CITATIONS

SEE PROFILE 


\title{
Multi-lead ECG Signal Analysis for Myocardial Infarction Detection and Localization through the Mapping of Grassmannian and Euclidean Features into a Common Hilbert Space
}

\author{
Panagiotis Barmpoutis ${ }^{1}$, Kosmas Dimitropoulos ${ }^{2}$, Anestis Apostolidis ${ }^{3}$, and Nikos Grammalidis ${ }^{*}$ \\ ${ }^{1}$ Department of Electrical and Electronic Engineering, Faculty of Engineering, Imperial College London, \\ SW7 2AZ, United Kingdom \\ ${ }^{2}$ Visual Computing Lab, Information Technologies Institute, Center for Research and Technology Hellas, \\ 57001, Greece \\ ${ }^{3}$ Department of Electrical and Computer Engineering, Faculty of Engineering, Aristotle University, \\ Hellas, 54124, Greece \\ \{p.barmpoutis@imperial.ac.uk, dimitrop@iti.gr, anestapostol@gmail.com, „ngramm@iti.gr\}
}

\begin{abstract}
Background and Objective: Electrocardiogram is commonly used as a diagnostic tool for the monitoring of cardiac health and the detection of possible heart diseases. However, the procedure followed for the diagnosis of heart abnormalities is time consuming and prone to human errors. Thus, the development of computer-aided techniques for the automatic analysis of electrocardiogram signals is of vital importance for the diagnosis and prevention of heart diseases. The most serious outcome of coronary heart disease is the myocardial infarction, i.e. the rapid and irreversible damage of cardiac muscles, which, if not diagnosed and treated in time, continues to damage further the myocardial structure and function. In this paper we propose a novel approach for the automatic detection and localization of myocardial infarction from multi-lead electrocardiogram signals.
\end{abstract}

Methods: The proposed method initially reshapes the multidimensional signal into a thirdorder tensor structure and subsequently extracts feature representations in both Euclidean and Grassmannian space. In addition, two different methods are proposed for the mapping of the two different feature representations into a common Hilbert space before the final classification of signals. The first approach is based on the mapping of both Grassmannian and Euclidean features in a Reproducing Kernel Hilbert Space (RKHS), while the second one 
attempts to initially apply Vector of Locally Aggregated Descriptors (VLAD) encoding directly to Grassmann manifold and then concatenate the two VLAD representations.

Results: For the evaluation of the proposed method, we have conducted extensive tests using a publicly available dataset, namely PTB Diagnostic ECG database, containing 549 multi-lead ECG data recordings from 290 subjects and from different diagnostic classes. The method provides an excellent detection rate of $100 \%$, and localization rate, i.e., $100 \%$ with the first fusion method and $99.7 \%$ with the second one.

Conclusions: The Experimental results presented in this paper show the superiority of the proposed methodology against a number of state-of-the-art approaches. The main advantage of the proposed approach is that it exploits better the intercorrelations between signals of different ECG leads, by extracting feature representations that lie in different geometrical spaces and contain complementary information with regard to the dynamics of signals.

Keywords: Electrocardiograms, Myocardial Infraction, Linear Dynamical Systems, Reproducing Kernel Hilbert Space

\section{Introduction}

Cardiovascular diseases (CVDs) are related to a number of factors preventing the flow of blood to heart or brain and are considered as the number one cause of death globally [1]. In severe conditions, deaths are due to the occlusion of the coronary artery caused by the rupturing of atherosclerotic plaques [2], something that constitutes the main pathogenesis for the majority of Myocardial Infarctions (MI). More specifically, the $\mathrm{MI}$ is one of the five main manifestations of coronary heart disease [1] and occurs when the blood flow decreases or stops to a part of the heart, causing damage to the heart muscle. Myocardial infarction can be recognized by clinical symptoms and features, including elevated values of biochemical markers (biomarkers) of myocardial necrosis and by imaging [3].

Recent advances in digital diagnostic technology can contribute significantly to the detection of even minor Ml events of cardiac patients effectively. Towards this direction, electrocardiogram (ECG) is an important tool for doctors, providing vital information with regard to the function and rhythm of human heart [4]. ECG signals are used for the diagnosis 
of cardiac symptoms and the detection of a variety of heart diseases like myocardial infarction (MI), cardiac dysrhythmias and pulmonary embolism. More specifically, Ml produces changes in the ECG signal appearing T waves abnormally high, longer than normal QT intervals and abnormal elevation of ST segment [5]. As the nature and amplitude of $P, Q, R, S, T$ waves in the ECG signals changes depending on the lead, the use of multiple ECG leads is required for an accurate analysis. Different types of MI can be observed in specific leads depending on the location of infarction in the myocardium.

Nowadays, to overcome time and reliable limitations of manual analysis of ECGs, several computer-aided signal processing methods have been proposed in order to detect and localize MI from ECG signals [6]. To accurately extract features from ECG signals many researchers have used discrete wavelet transform (DWT) techniques [7], [8], as DWT has been proved an efficient tool for the analysis of this kind of signals. More specifically, Zhao and Zhang [9] proposed the use of wavelet transform and support vector machines, while for the estimation of subtle changes of ECG signals, Jayachandran [10] utilized the multiresolution properties of DWT to identify characteristic points in ECG signal and computed the entropy in the wavelet domain. On the other hand, other researchers [11] have proposed the use of Fourier harmonic phases of the ECG data which is advantageous in terms of computational simplicity.

However, the majority of the aforementioned techniques is based on the analysis of single-lead ECGs instead of multi-lead ECG (MECG) signals. In [12], the authors presented an automatic detection and localization approach of myocardial infarction (MI) using Knearest neighbor (KNN) classifier. Specifically, time domain features of each beat in the ECG signal, which are indicative of $\mathrm{Ml}$, such as $\mathrm{T}$ wave amplitude, $\mathrm{Q}$ wave and ST level deviation are extracted from 12 leads ECG. In another research work, Sharma, et al. introduced a novel technique based on a multiscale energy and eigenspace (MEES) approach for the detection and localization of MI [13]. Furthermore, Padhy and Dandapat proposed a method for MI detection and localization where higher-order singular value decomposition was applied to a third-order MECG tensor for dimensionality reduction [14], while for the detection and localization of MI a multi-class SVM classifier was used. 
Recently, deep learning networks have been employed in the automated classification of ECG signals and detection of numerous heart diseases [15], [16]. For MI detection, Acharya et al., [5] used a 11-layer deep CNN algorithm for the detection of MI using ECG multi-lead signals with and without noise. Furthermore, Liu, et al., [17] used a deep convolution neural network (CNN) using electrocardiogram (ECG) signal from the lead II and taking 3-second signal segments as input. Although these methods don't rely on the extraction of handcrafted features, the training of complex deep learning networks requires the creation of large datasets for the accurate definition of their parameters.

In this paper, we propose a novel approach for MI detection and localization that exploits better the intercorrelations between signals of different ECG leads by extracting feature representations that lie in different geometrical spaces and contain complementary information with regard to the dynamics of signals. In addition, we propose two different fusion approaches for mapping the extracted feature representations into a common Hilbert space. More specifically, the contributions of this work can be summarized in the following aspects:

- We introduce a novel methodology for automated myocardial infarction detection and localization, aiming to improve classification accuracy by fusing different feature representations with complementary information into a common Hilbert space. The proposed methodology has been benchmarked in a popular publicly available dataset with favorable results.

- In order to exploit the hidden beat and lead correlations, we propose the modeling of multi-lead ECG signals through a higher-order Linear Dynamical System (LDS) and the projection of LDS parameters to a Grassmann manifold.

- Moreover, we extract feature representations in the Euclidean space by encoding multi-lead ECG signals as vectors of locally aggregated descriptors (VLAD) after a dyadic discrete wavelet transform and a subsequent multiscale higher-order SVD analysis on sub-band tensors.

- Finally, we propose two fusion approaches for mapping the extracted feature representations in a common Hilbert space. The first approach is based on the mapping of both Grassmannian and Euclidean features in a Reproducing Kernel Hilbert Space (RKHS), while the second one attempts to apply VLAD encoding 
directly to Grassmann manifold and then concatenate the two VLAD representations. Both approaches are generic and can be easily applied to various application fields.

The remainder of this paper is organized as follows: Section 2 presents the proposed methodology including data preprocessing, ECG signals modeling, fusion of feature representations and classification. Subsequently, the dataset and experimental results are discussed in Section 3, while finally conclusions are drawn in Section 4.

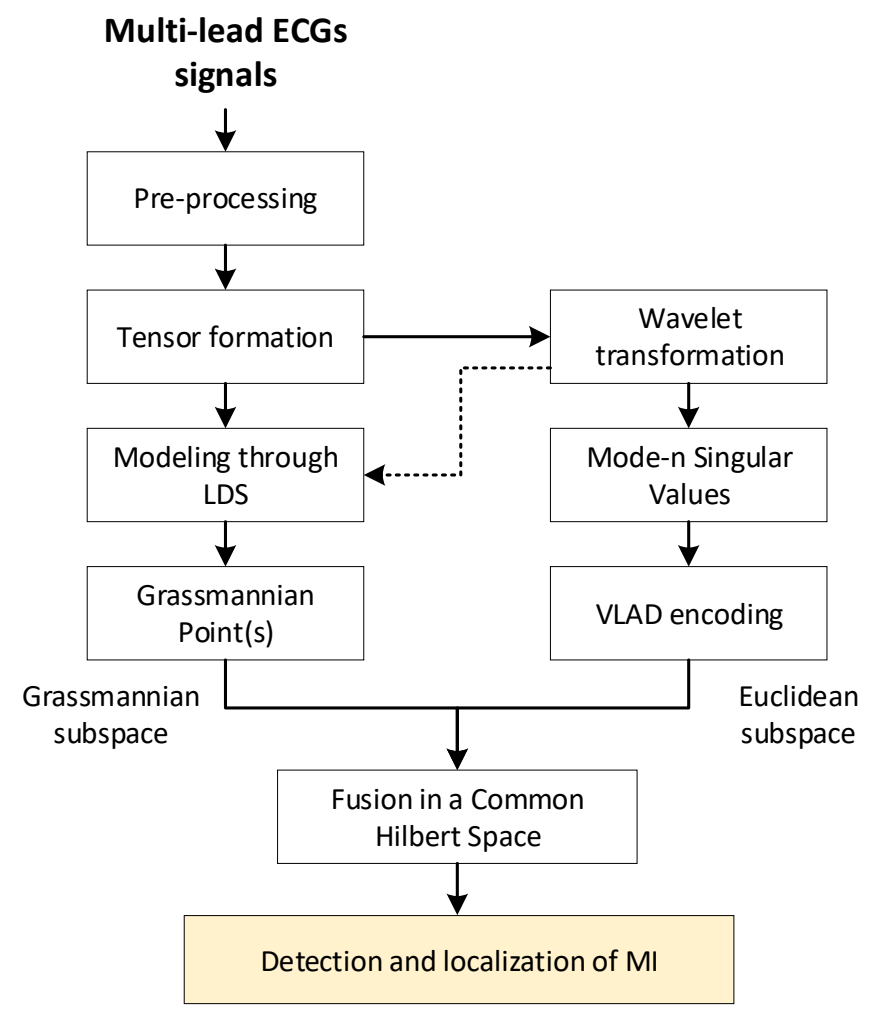

Figure 1. - The proposed methodology.

\section{Methods}

The overall structure of the proposed methodology for the classification and localization of MI is shown in Figure 1. More specifically, multi-lead ECG signals are initial pre-processed and reshaped into a third-order tensor structure, while, subsequently, Euclidean and Grassmannian feature representations are extracted and fused in a common Hilbert space. In the case of Euclidean features, VLAD encoding is applied after a dyadic discrete wavelet transform and a subsequent multiscale higher-order SVD analysis on sub-band tensors. On the other hand, Grassmannian features, i.e., points on Grassmann manifold, are extracted by feeding the third-order tensor structure to a higher-order linear dynamical system. 
Alternatively, we can also model the sub-band tensors of wavelet transform using higherorder linear dynamical systems to create multiple Grassmannian subspaces (this issue is discussed in detail in section 2.4.2). In the last stage of the proposed approach, the two different feature representations are mapped into a common Hilbert space for the detection and localization of MI.

\subsection{Data preprocessing and tensor ECG formation}

In this first preprocessing step, we attempt to bring the leads of ECG to their isoelectric levels by passing each ECG signal through a digital Butterworth high-pass filter with a cut-off frequency of $0.5 \mathrm{~Hz}$, and a "Zero-phase forward and reverse digital filter", as proposed in [18]. Additionally, R-peak detection and period normalization is performed, while subsequently each lead signal is segmented and normalized to the number of beat periods. After the preprocessing of the recorded signals, the multi-lead ECG data is reshaped into a third-order tensor structure. More specifically, this data is represented as $Y \in \mathbb{R}^{l \times b \times s}$, where the dimensions $l, b$, and $s$ are respectively the number of leads, heart beats, and consecutive samples of normalized heartbeat. The horizontal slices of $Y$ represent each ECG lead, and each vector of a horizontal slice represents consecutive beats of a lead [14].

\subsection{Mapping of ECGs signals into Grassmann Manifold}

The output of an ECG signal indicates the electrical activity generated by the heart as a function of time and it is a near-periodic signal for a specific time. In a multi-lead ECG the leads refer to imaginary lines between two ECG electrodes. To exploit this information, in this paper we attempt to model the interdependent 12-lead signals and beats of ECGs using linear dynamical systems (LDSs). A linear dynamical system is associated with a first order ARMA process with white zero mean IID Gaussian input and for this reason LDSs are also known as linear Gaussian state-space models. In general, LDS models attempt to associate the output of the system, i.e., the observation, with a linear function of a state variable, while in each time instant, the state variable depends linearly on the state of the previous time instant. Both state and output noise are zero-mean normally distributed random variables and apart from the output of the system, all other variables (state and noise variables) are hidden. The adopted system is described by the following equations: 


$$
\begin{aligned}
& x(t+1)=A x(t)+B v(t) \\
& y(t)=\bar{y}+C x(t)+w(t)
\end{aligned}
$$

where $x \in R^{n}$ is the hidden state process, $y \in \mathbb{R}^{d}$ is the observed data, $A \in \mathbb{R}^{n \times n}$ is the transition matrix of the hidden state and $C \in \mathbb{R}^{d \times n}$ is the mapping matrix of the hidden state to the output of the system. The quantities $w(t)$ and $B v(t)$ are the measurement and process noise respectively, with $w(t) \sim N(O, R)$ and $B v(t) \sim N(0, Q)$, while $\bar{y} \in \mathbb{R}^{d}$ is the mean value of the observation data. The extracted tuple LDS descriptor, $M=(A, C)$, models both the appearance and dynamics of the observation data, represented by $C$ and $A$, respectively. The descriptor's parameters, $A$ and $C$, can be estimated through a suboptimal method initially proposed by Doretto et. al [19].

However, in our case the multi-lead ECG signal is represented by the third-order tensor $Y$. To this end, we decompose the ECG formation $Y$ using a higher order singular value decomposition:

$$
Y=S \times{ }_{1} U_{(1)} \times_{2} U_{(2)} \times_{3} U_{(3)}
$$

where $S \in \mathbb{R}^{l \times b \times s}$ is the core tensor, while $U_{(1)} \in \mathbb{R}^{l \times l}, U_{(2)} \in \mathbb{R}^{b \times b}$ and $U_{(3)} \in \mathbb{R}^{s \times s}$ are orthogonal matrices containing the orthonormal vectors spanning the column space of the matrix and $x_{j}$ denotes the $j$-mode product between a tensor and a matrix. Since the columns of the mapping matrix $C$ of the stochastic process need to be orthonormal, we can consider $C=U_{(3)}$ and

$$
X=S \times{ }_{1} U_{(1)} \times{ }_{2} U_{(2)}
$$

Then, the transition matrix A can be estimated using least squares [20] as follows:

$$
A=X_{2} X_{1}^{T}\left(X_{1} X_{1}^{T}\right)^{-1}
$$

where $X_{1}=[x(2), x(3), \ldots, x(t)]$ and $X_{2}=[x(1), x(2), \ldots, x(t-1)]$.

Furthermore, to improve the stability of the dynamical system (i.e., to estimate the stabilized transition matrix $A$ ), we obtain an approximate solution, based on a convex optimization technique [21], by solving the following quadratic problem:

$$
\begin{gathered}
\text { minimize } a P a-2 q^{T} a+r \\
\text { subject to } g^{T} a \leq 1
\end{gathered}
$$


where $a=\operatorname{vec}(A), q=\operatorname{vec}\left(X_{1} X_{2}^{T}\right), \quad r=\operatorname{tr}\left(X_{2}^{T} X_{2}\right)$ and $P=I \otimes\left(X_{1}^{T} X_{1}\right), \quad I \quad$ is the identity matrix, $\operatorname{tr}(\cdot)$ indicates the trace of a matrix and $\operatorname{vec}(\cdot)$ operator converts a matrix to vector and $\otimes$ denotes the Kronecker product. Also, $g=\operatorname{vec}\left(u_{1} v_{1}^{T}\right)$ where vectors $u_{1}$ and $v_{1}^{T}$ correspond to the first eigenvalue of the transition matrix $A$.

Having modeled each ECG signal using a higher-order linear dynamical system approach, our next step is to represent the parameters of each dynamical system, $M=(A, C)$, as a point on the space of the extracted descriptors. Towards this end, we initially estimate the finite observability matrix of each dynamical system, $O_{m}^{T}(M)=$ $\left[C^{T},(C A)^{T},\left(C A^{2}\right)^{T}, \ldots,,\left(C A^{m-1}\right)^{T}\right]$ and then, we apply a Gram-Schmidt othonormalization [22] procedure, i.e., $O_{m}^{T}=G R$, in order to represent each descriptor $M$ as a point, $G \in \mathbb{R}^{m \times T \times 3}$, on the Grassmann manifold (in our experiments we set the size $m$ of the observability matrix equal to 3 , while $T$ is the number of samples).

\subsection{Modelling of ECG signal using locally aggregated descriptors}

In this section, we propose the representation of ECG signals, i.e., the third-order tensor $Y$, as a Vector of Locally Aggregated Descriptors (VLAD). The extracted VLAD descriptor can be considered intrinsically as Euclidean, as it encodes the features' distribution in their native vector space. More specifically, we initially apply a dyadic discrete Wavelet Transform (with Daubechies $9 / 7$ Biorthogonal wavelet filters as mother wavelet) on every vector $Y(i, j,:)$ of the tensor $Y \in \mathbb{R}^{l \times b \times s}$ where $i=1, \ldots, l$ and $j=1, \ldots, b$. This transformation results $2 \times L$ sub-band tensors ( $L$ is the number of levels and depends on the sampling frequency of the signal) comprising of one approximation $A_{L} \in r^{l \times b \times s_{A}}$, with $s_{A}=s / 2^{\wedge} L$, and $L$ number of details $D_{k}$ (where $k=1, \ldots, L)$ sub-band tensors with dimensions $l \times b \times s_{k}$, with $s_{k}=s / 2^{\wedge} k$ [14]. Subsequently, each sub-band tensor is decomposed according to equation (3) and a feature vector is formed by the concatenation of mode- $n$ singular values $\sigma$ (in our case $n$ is equal to 3) of all extracted core tensors $S$. In particular, for the 3 modes of each sub-band tensor $A_{L}$ and $D_{k}$, we form the corresponding feature vectors:

$$
x_{L}^{A}=\left[\sigma_{1}^{(1)}, \ldots, \sigma_{l}^{(1)}, \sigma_{1}^{(2)}, \ldots, \sigma_{b}^{(2)}, \ldots, \sigma_{1}^{(3)}, \ldots, \sigma_{s_{A}}^{(3)}\right]
$$

and 


$$
y_{k}^{D}=\left[\hat{\sigma}_{1}^{(1)}, \ldots \hat{\sigma}_{1}^{(1)}, \hat{\sigma}_{1}^{(2)}, \ldots \hat{\sigma}_{\mathrm{b}}^{(2)} \ldots, \hat{\sigma}_{1}^{(3)}, \ldots \hat{\sigma}_{s_{k}}^{(3)}\right]
$$

and then, we concatenate the individual features to form the final feature vector as follows:

$$
v=\left[x_{\mathrm{L}}^{A}, y_{\mathrm{L},}^{D}, y_{L-1}^{D}, \ldots, y_{1}^{D}\right]
$$

In our experiments we extracted the singular values of sub-band tensors $A_{7}, D_{7}, D_{6}, D_{5}$ and $D_{4}$ and used the first three singular values of each unfolded submatrix for the construction of feature vectors in equations (8) and (9). These sub-bands contain 'PQRST' segmented information, while the rest do not contain any meaningful information [14].

Finally, for the modelling of each ECG signal, we apply VLAD encoding, which is considered as a simplified coding scheme of the earlier Fisher Vector (FV) representation and has shown to outperform histogram representations in bag of features approaches [23], [24]. More specifically, we consider a codebook, $\left\{m_{i}\right\}_{i=1}^{r}=\left\{m_{1}, m_{2}, \ldots, m_{r}\right\}$, with $r$ visual words and local descriptors $v$, where each descriptor is associated to its nearest codeword $m_{i}=N N\left(v_{j}\right)$. The VLAD descriptor, $V$, is created by concatenating the $r$ local difference vectors $\left\{u_{i}\right\}_{i=1}^{r}$ corresponding to differences $v_{j}-m_{i}$, with $m_{i}=N N\left(v_{j}\right)$, where $v_{j}$ are the descriptors associated with codeword $i$, with $i=1, \ldots, r$.

$$
\bar{V}=\left\{u_{i}\right\}_{i=1}^{r}=\left\{u_{1}, \ldots, u_{r}\right\}
$$

or

$$
\bar{V}=\left\{\sum_{\substack{v_{j} \text { such that } \\
m_{1}=N N\left(v_{j}\right)}}\left(v_{j}-m_{1}\right), \ldots, \sum_{\begin{array}{c}
v_{j} \text { such that } \\
m_{r}=N N\left(v_{j}\right)
\end{array}}\left(v_{j}-m_{r}\right)\right\}
$$

while the final VLAD representation is determined by the L2-normalization of vector $\bar{V}$ :

$$
\bar{V}_{\text {Euclidean }}=\bar{V} /\|\bar{V}\|_{2}
$$

\subsection{Fusion of feature representations in a common space}

\subsubsection{Fusion through the mapping of data in a Reproducing Kernel Hilbert Space}

To fuse the extracted feature representations, we propose in this section their mapping into a common Hilbert space $H$ (it is defined as proposed-1). Our main problem here stems from the fact that the two feature representations, i.e., Grassmannian points and VLAD encodings, lie in different geometrical spaces. More specifically, in the first case we have points in the non- 
Euclidean space of the dynamical model, known as Grassmann manifold, which is a quotient of the special orthogonal group $S O(n)$, i.e., the subset of all orthogonal matrices with determinant equal to +1 (this simply means that we can extend the notion of tangent spaces, geodesics etc. from the base manifold $S O(n)$ to the quotient space of Grassmann manifold). On the other hand, the second feature representation is a VLAD descriptor, which lies in Euclidean space (Figure 2).

To address the problem, we attempt to transform the two feature representations into a common Hilbert space using two kernel functions, $\varphi_{G}: G \rightarrow H$ for the Grassmann manifold and $\varphi_{E}: \mathbb{R}^{r} \rightarrow H$ for the Euclidean space. In the first case, the Grassmannian kernel $k_{G}\left(g_{1}, g_{2}\right)$, which shows the similiarity between two Grassmannian points $g_{1}$ and $g_{2}$ is estimated using the inverse exponential map on the Grassmann manifold:

$$
k_{G}\left(g_{1}, g_{2}\right)=d_{G}\left(g_{1}, g_{2}\right)=\left\|\exp _{g_{2}}^{-1} g_{1}\right\|_{F}
$$

where $\|\cdot\|_{F}$ is the matrix Frobenius norm. For estimating the inverse exponential map, we first need to compute the orthogonal completion $O_{r}$ of $g_{1}$ and then the thin CS decomposition of matrix $O_{r}^{T} g_{2}$ to find the direction matrix that specifies the direction and speed of geodesic flow [25]. On the other hand, for the Euclidean space, we can simply apply a Radial Basis Function (RBF) kernel for two feature vectors $x_{1}$ and $x_{2}$ :

$$
k_{E}\left(x_{1}, x_{2}\right)=\exp \left(-\frac{\left\|x_{1}-x_{2}\right\|^{2}}{2 \sigma^{2}}\right)
$$

Using equations (14) and (15) as similarity metrics in Grassmannian and Euclidean space respectively, we can easily estimate the elements of kernel matrices $K_{G}$ and $K_{E}$. To improve the robustness and the discriminative ability of the method, we create kernels of equal size, i.e., $K_{G}, K_{E} \in \mathbb{R}^{M x M}$, with $M=k * c$, where $c$ is the number of classes, while $k$ is the number of the most representative samples in each class. For the Euclidean space, we apply a simple $k$ means algorithm, while for the Grassmannian manifold, we select the most representative Grassmannian Points, using as a distance between two points on the manifold, the similarity metric of equation (14). To this end, we apply a $k$-medoids classification approach considering as medoid, the local minimizer of function $\mathrm{F}$ :

$$
F\left(m_{k}\right)=\frac{1}{n_{G}} \sum_{i=1}^{n_{G}} d_{G}\left(m_{k}, g_{i}\right)
$$


where $n_{G}$ indicates the total number of Grassmannian points $g_{i}$ in a medoid $m_{k}$ and $d_{G}(\cdot)$ denotes the distance between two Grassmannian points (see equation (14)). Having defined the $M$ most representative Grassmannian points among the existing ones, we estimate the Grassmannian kernel matrix as $K_{G_{i, j}}=k_{G}\left(g_{i}, g_{j}\right)$, with $i, j=1,2, \ldots M$. Similarly, each element of the Euclidean kernel matrix is defined as $K_{E_{i, j}}=k_{E}\left(x_{i}, x_{j}\right)$ for each $i, j \in[1, M]$. Subsequently, we estimate the common kernel matrix for the two subspaces as $K_{E G}=K_{E}{ }^{\circ} K_{G}$, where $K_{E G} \in \mathbb{R}^{M x M}$ and $\left\langle\left\langle^{\circ}\right\rangle\right.$ is the Handamard product of kernel functions.

Finally, to classify the ECG signals we apply a sparse representation using the following equation according to [26], which enables us to map the input signal $y$ to the Hilbert space of a sparse representation $a_{s}$ :

$$
\min _{a}\left\|y-a_{s} C\right\|_{2}^{2}+\lambda\left\|a_{s}\right\|_{1}
$$

where $\mathrm{y}=\Sigma^{1 / 2} U^{T} K_{E G}$ and $\mathrm{C}=\Sigma^{1 / 2} U^{T}$, with $U \Sigma V=K_{E G}$ [27].

For the detection and localization of MI, ECG signals corresponding to myocardial infarction cases were initially discriminated from those of health controls and then they were classified in the following categories: anterior (AMI), antero-lateral (ALMI), antero-septal (ASMI), inferior (IMI) and infero-lateral (ILMI). To this end, each ECG signal represented by a set of sparse coefficients is classified to a class $i=1 \ldots N$, whose training samples provide the best reconstruction of it. Specifically, the classification is performed by assigning each multilead ECG signal $x$ to the class minimizing the following residual:

$$
\text { Class }(i)=\arg \min _{i}\left\|x-\delta_{i}\left(a_{s}\right) C\right\|
$$

where $\delta_{i}$ sets to zero the coefficients of $a_{s}$ that do not correspond to class $i$. The estimation of the sparse representations of ECG signals is achieved using the SPAMS toolbox of Matlab. 


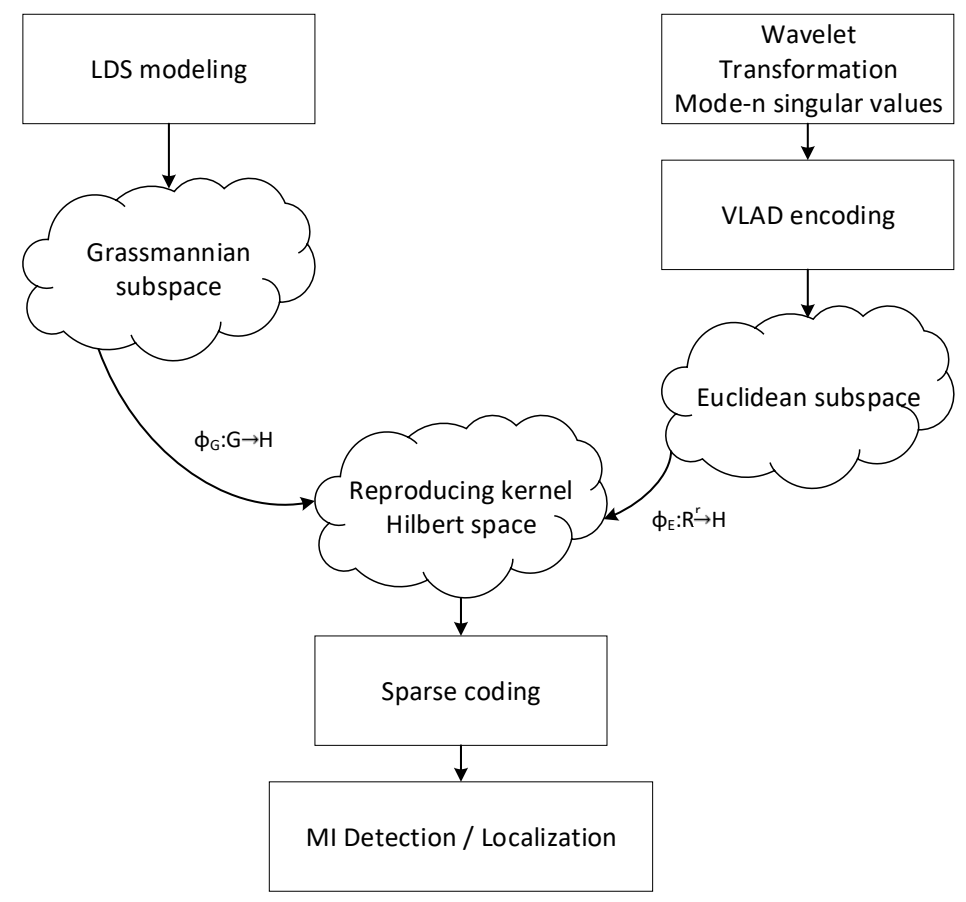

Figure 2. Detection accuracy rates for proposed-1 fusion approach using different kernel functions for mapping Euclidean data into a common Hilbert space

\subsubsection{Fusion in a Hilbert space through VLAD encoding}

In this second fusion approach, we attempt to apply VLAD encoding on Grassmann manifold, as in the case of the Euclidean space, and then concatenate the two VLAD representations to form a joint representation for the two spaces (it is defined as proposed-2). In other words, we use VLAD encoding as a means to map our features into a common space.

In contrast with the previous approach, in this case, we divide each signal into overlapping equally-sized elementary signals (using a sliding window of a constant size $W$ ) that are modeled by a linear dynamical system. In this way, each ECG signal is finally represented as a set of points on the Grassmann manifold instead of a single point. Subsequently, we apply a Karcher mean algorithm [28] to estimate the codewords $m_{i}$ in equation (12). We re-identify the members $G_{j}$ of each class, i.e., $m_{i}=N N\left(G_{j}\right)$, using the dissimilarity metric defined in equation (14). Hence, the VLAD encoding of an ECG signal on the Grassmann manifold for a codebook of $q$ representative words, $\left\{m_{i}\right\}_{i=1}^{q}$, can be defined as:

$$
\bar{V}_{\text {Grassmannian }}=\frac{\bar{V}}{\|\bar{V}\|_{2}}=
$$




$$
=\frac{1}{\|\bar{V}\|}\left\{\sum_{\begin{array}{c}
G_{j} \text { such that } \\
\text { the Karcermean } \\
m_{1}=N N\left(G_{j}\right)
\end{array}}\left\|\exp _{m_{1}}^{-1} G_{j}\right\|_{F}, \ldots, \sum_{\begin{array}{c}
G_{j} \text { such that } \\
\text { the Karcer mean } \\
m_{q}=N N\left(G_{j}\right)
\end{array}}\left\|\exp _{m_{q}}^{-1} G_{j}\right\|_{F}\right\}
$$

For the final classification of an ECG signal to one of the five classes (localization), i.e., anterior (AMI), antero-lateral (ALMI), antero-septal (ASMI), inferior (IMI) and infero-lateral (ILMI), we concatenate the two VLAD encodings, i.e., $\bar{V}_{E C G}=\left[\bar{V}_{\text {Euclidean }} \bar{V}_{\text {Grassmannian }}\right]$, to form a joint feature representation and finally use a simple SVM classifier. We have to note here that when sub-band tensors of wavelet transform are modeled using higher-order linear dynamical systems, the system creates multiple Grassmannian subspaces. In our experiments, we used in total five sub-bands, $A_{7}, D_{4}, D_{5}, D_{6}$ and $D_{7}$ and therefore in this case the final vector $\bar{V}_{E C G}$ was produced by the concatenation of one Euclidean with six

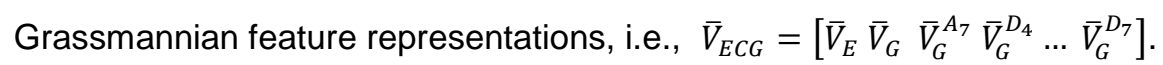

\section{Results}

For the evaluation of the proposed method we conducted extensive tests using a publicly available dataset, namely PTB Diagnostic ECG database [29], containing multi-lead ECG data. Each record in the dataset includes 15 simultaneously measured signals, i.e., the conventional 12 leads as shown in Figure 3 (i, ii, iii, avr, avl, avf, v1, v2, v3, v4, v5, v6) together with the 3 Frank lead ECGs (vx, vy, vz) (in our experiments the 3 Frank lead ECGs were not used, Figure 3). Figure 4 shows a 3D representation of a beat period of an ECG signal of the PTB Diagnostic database. Each signal is digitized at 1000 samples per second. More specifically, the dataset contains in total 549 records of 290 subjects from different diagnostic classes: Myocardial infarction (148), Cardiomyopathy/Heart failure (18), Bundle branch block (15), Dysrhythmia (14), Myocardial hypertrophy (7), Valvular heart disease (6), Myocarditis (4), Miscellaneous (4), Healthy controls (52). In the case of MI patients, the location of the infarction in the myocardium is classified in different categories [30]. Nevertheless, in our experiments five groups of these have been considered (anterior (AMI), antero-lateral (ALMI), antero-septal (ASMI), inferior (IMI) and infero-lateral (ILMI)). The records of these categories are 47 from AMI, 43 from ALMI, 77 from ASMI, 89 from IMI and 
56 from ILMI. The other 56 records from Ml subjects correspond to other groups of MI location, but these groups have a very limited number of ECG records to be used for training the classifier.

The goal of this experimental evaluation is three-fold. Firstly, we aim to define the optimal parameters for the two proposed fusion approaches. Secondly, a detailed experimental evaluation for the contribution of each extracted feature and fusion approach is performed estimating the $\mathrm{MI}$ detection and localization rates. Finally, in order to validate the efficiency of the proposed method, we compared its detection and localization rates with those of various state-of-the-art approaches using the same dataset.

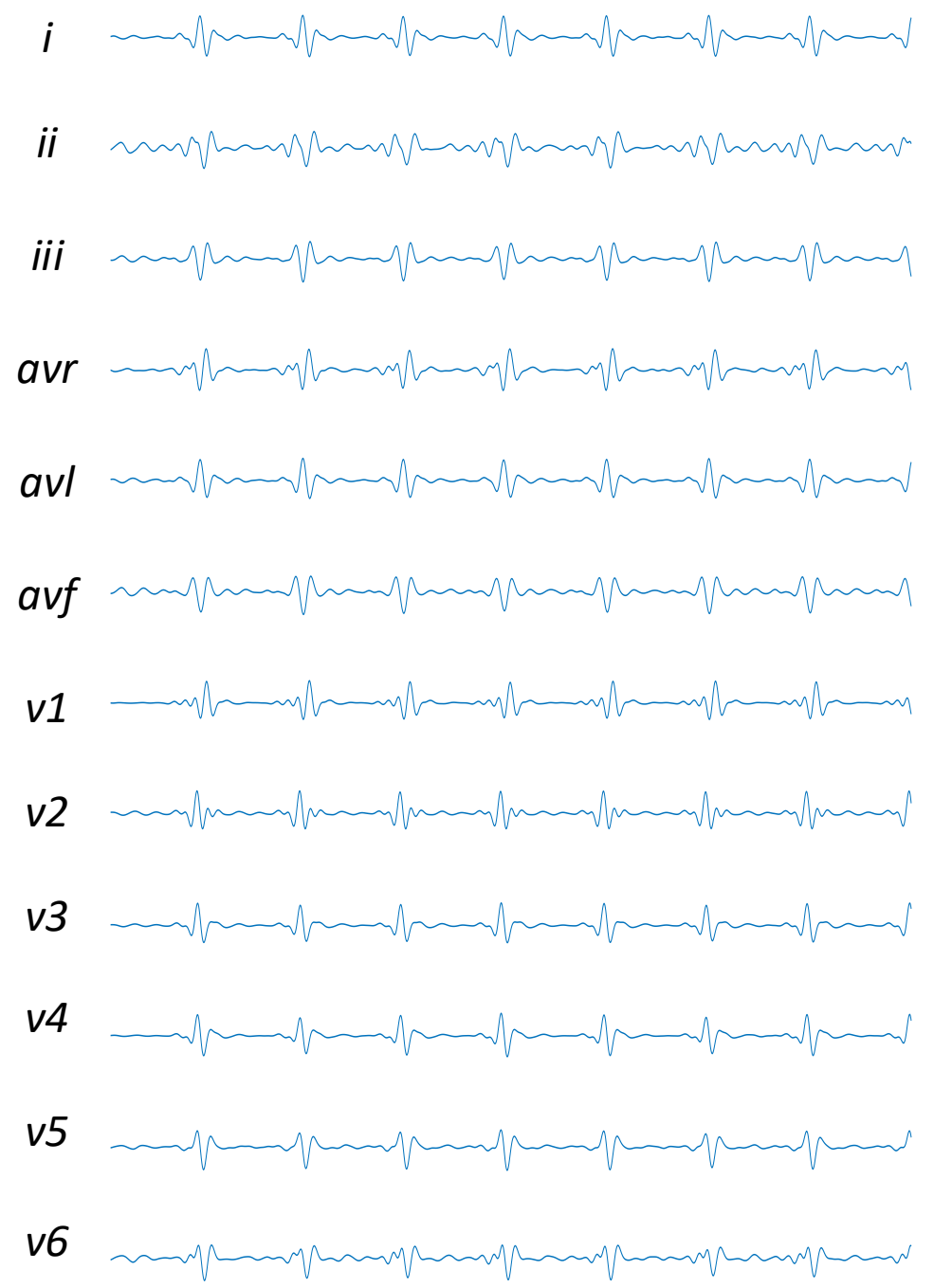

Figure 3. The 12 leads of an ECG signal of the PTB Diagnostic database 


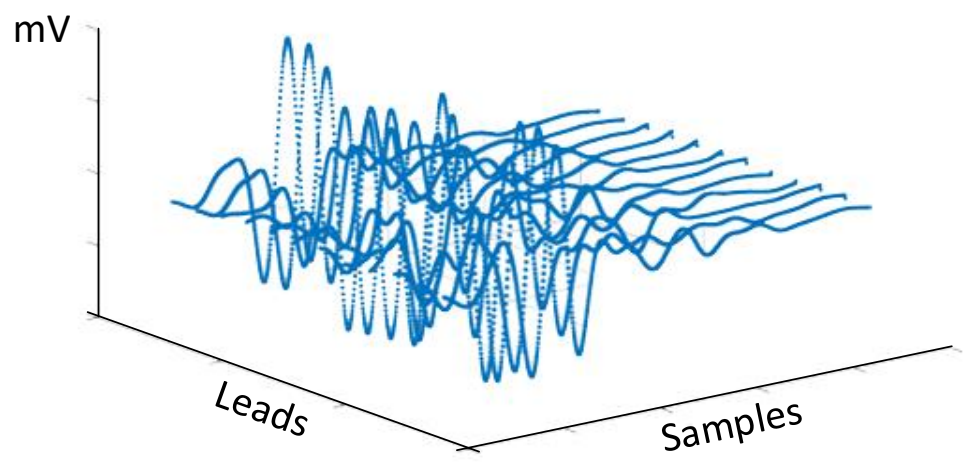

Figure 4. A beat period of an ECG signal of the PTB Diagnostic database

\subsection{Estimating the optimal parameters}

The first evaluation phase concerns the selection of the optimal parameters for both fusion approaches in order to achieve the best detection rates. Initially, we carried out experiments in order to define the most appropriate kernel function for the mapping of Euclidean data into the Reproducing Kernel Hilbert Space (proposed-1 approach). In particular, we experimented using three kernel functions, namely radial basis function (RBF), polynomial and exponential chi-square distance. Figure 5 presents the classification rates for each kernel function when they are applied for the mapping of Euclidean data into a common Hilbert Space. As we can easily see, the best classification rate $(100 \%)$ is achieved by using the RBF kernel function, while the polynomial and chi-square kernels provide lower detection rates of $99.6 \%$ and $93.8 \%$, respectively.

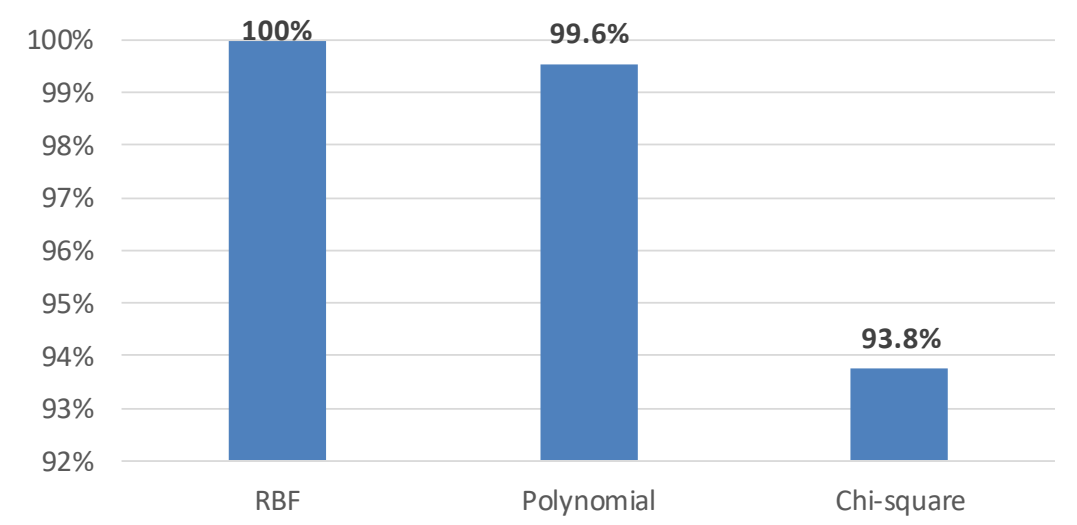

Figure 5. Detection accuracy rates for proposed-1 fusion approach using different kernel functions for mapping Euclidean data into a common Hilbert space

On the other hand, for the second fusion approach (proposed-2), we attempt to find the most appropriate sliding window size $W$ for dividing the multi-dimensional signal into 
overlapping equally sized elementary signals. This approach allows us to represent each signal as a set of points on the Grassmann manifold instead of a single point. Towards this end, we carried out experiments using five different window sizes $(5,10,15,20$ and 25 length) for creating the elementary equally sized signals. As seen in Figure 6, the best classification rate is achieved by setting the window size equal to 20 , yielding a detection rate of $95.83 \%$ (this detection rate refers to the VLAD encoding $\bar{V}_{G}$ and not to the concatenated $\bar{V}_{E C G}$ vector). It is worth mentioning that when small signal sizes are used, the detection rates decrease, apparently due to the lack of sufficient information in each sub-signal, while the detection rate for signal sizes larger than 20 also seems to decrease.

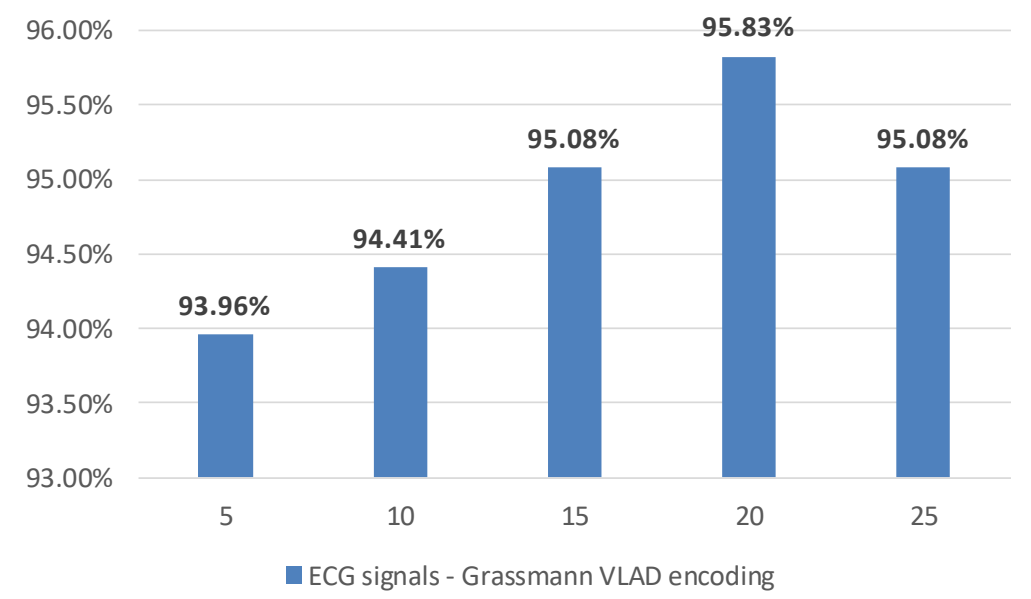

Figure 6. Detection rates of Grassmann VLAD encoding $\bar{V}_{G}$ (proposed-2 approach) using different window sizes $W$.

\subsection{Contribution of different feature representations to the detection of myocardial}

\section{infraction}

In this subsection, we elaborate a more detailed analysis in order to evaluate the contribution of different feature representations to the MI detection process. Specifically, we analyze the contribution of four different feature representations: a) The representation of ECG signal as a single Grassmann point (proposed-1 approach). b) The VLAD encoding representation $\bar{V}_{E}$ of the ECG signal after the dyadic discrete wavelet transform and the subsequent multiscale higher-order SVD analysis on sub-band tensors (proposed-1 and proposed-2 approach). c) The VLAD encoding representation on the Grassmann manifold (proposed-2 approach) using a single Grassmann subspace i.e., $\bar{V}_{G}$ and d) The concatenated VLAD encoding representation of the five sub-bands, $A_{7}, D_{4}, D_{5}, D_{6}$ and $D_{7}$ (proposed-2 approach). 
For the classification of the ECG signals, in the first case, i.e., Grassmannian subspace, we used as a similarity metric the distance between two Grassmannian points, as defined in equation (14), while in the cases of VLAD encodings we used a standard SVM classifier. The experimental results in Figure 7 show that VLAD encoding on Grassmann manifold, i.e., $\bar{V}_{G}$, achieves the best results, with a detection rate of $95.8 \%$ against $94.6 \%$, 92.3\% and $91.3 \%$ for the VLAD encoding representation $\bar{V}_{E}$, the Grassmann feature representation and the concatenated VLAD vector, respectively. In the next section, we show that by mapping these features to a common Hilbert space, we can further improve the classification accuracy of individual features.

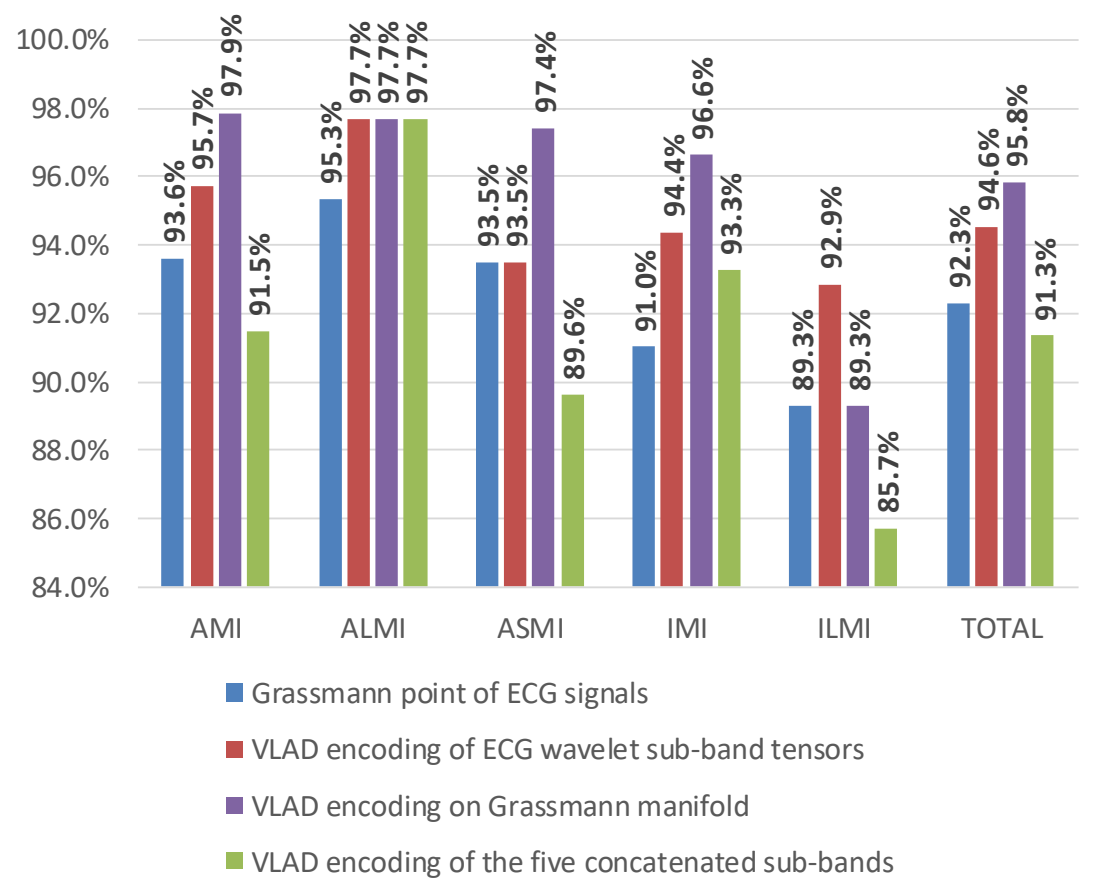

Figure 7. MI detection rates for the individual feature representations.

\subsection{Comparison of fusion approaches}

In this subsection, we aim to evaluate the effect of the two proposed fusion approaches to the detection and localization of MI. In the first approach (proposed-1), we use kernel functions to map the Grassmann feature representation and VLAD encoding $\bar{V}_{E}$ into a common Hilbert space and then apply sparse coding, while for the second approach (proposed-2) we create two concatenated VLAD vectors $\bar{V}_{E C G}=\left[\begin{array}{llll}\bar{V}_{E} & \bar{V}_{G} & \bar{V}_{G}^{A_{7}} & \bar{V}_{G}^{D_{4}} \ldots \bar{V}_{G}^{D_{7}}\end{array}\right]$ and $\bar{V}_{E C G}=\left[\bar{V}_{E} \bar{V}_{G}\right]$ and use a standard SVM classifier. 
As we can see in Figure 8, all approaches provide excellent detection rates and can easily distinguish MI cases from those of healthy controls. Additionally, considering the five types of MI, namely AMI, ALMI, ASMI, IMI and ILMI, the localization rates are $100 \%, 99.7 \%$ and $98.4 \%$ for proposed-1, proposed-2 with $\bar{V}_{E C G}=\left[\bar{V}_{E} \bar{V}_{G} \bar{V}_{G}^{A_{7}} \bar{V}_{G}^{D_{4}} \ldots \bar{V}_{G}^{D_{7}}\right]$ and proposed-2 with $\bar{V}_{E C G}=\left[\bar{V}_{E} \bar{V}_{G}\right]$, respectively. It is also worth mentioning that although the feature representation of VLAD encoding $\bar{V}_{G}$ on Grassmann manifold provides better results than a simple Grassmann feature, the fusion through a Reproducing Kernel Hilbert Space achieves better results in the case of localization. In addition, the accuracy rates of both approaches in Figure 8 make evident that the individual feature representations contain complementary information and therefore the detection accuracy after fusion is increased.

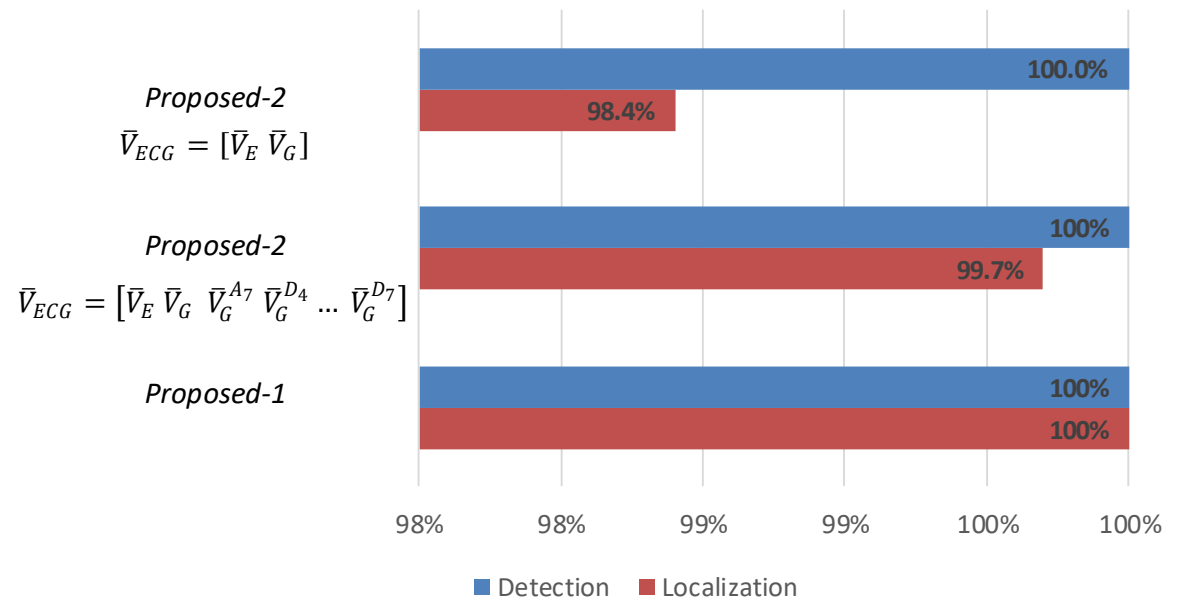

Figure 8. Detection and localization accuracy rates using the proposed fusion schemes

\subsection{Comparison with state-of-the-art approaches}

In this last section, we present a comparative analysis of the proposed method against a number of state-of-the-art approaches. More specifically, we compare the Sensitivity, Specificity and Accuracy rates of the proposed method (Table I) against those of ten state-ofthe-art approaches that have been used in the past for the detection and localization of $\mathrm{MI}$ on PTB Diagnostic ECG database.

To ensure a fair comparison, we adopted the same experimental protocol followed in [14]. The experimental results in Table I show that the proposed method (both proposed-1 and proposed-2 approaches) outperform all other methods achieving improvements up to 
$0.7 \%$ in detection accuracy from [17] and up to $1.9 \%, 1.3 \%, 0.4 \%$ and $1.2 \%$ in localization accuracies from [14], [31], [13] and [12], respectively.

TABLE I. Detection and localization comparison results

\begin{tabular}{|c|c|c|c|c|}
\hline & \multicolumn{3}{|c|}{ Detection } & Localization \\
\hline & Sen & Spe & Acc & Acc \\
\hline Proposed-1 & $100 \%$ & $100 \%$ & $100 \%$ & $100 \%$ \\
\hline Proposed-2 & $100 \%$ & $100 \%$ & $100 \%$ & $99.7 \%$ \\
\hline Liu et al. (2018) - [17] & $99.8 \%$ & $97.4 \%$ & $99.3 \%$ & NA \\
\hline Acharya et al. (2017) - [5] & $95.5 \%$ & $94.2 \%$ & $95.2 \%$ & NA \\
\hline Padhy et al. (2017) - [14] & $94.6 \%$ & $96 \%$ & $95.3 \%$ & $98.1 \%$ \\
\hline Sadhukhan et al. (2017) - [11] & $98.2 \%$ & $97.4 \%$ & $97.4 \%$ & NA \\
\hline Acharya et al. (2016) - [31] & $99.5 \%$ & $96.3 \%$ & $98.8 \%$ & $98.7 \%$ \\
\hline Sharma et al. (2015) - [13] & $93 \%$ & $99 \%$ & $96 \%$ & $99.6 \%$ \\
\hline Arif et al. (2012) - [12] & $99.97 \%$ & $99.9 \%$ & NA & $98.8 \%$ \\
\hline Sun et al. (2012) - [3] & $92.6 \%$ & $82.4 \%$ & NA & $76.6 \%$ \\
\hline Jayachandran et al. (2010) - [10] & NA & NA & $95.9 \%$ & NA \\
\hline Nugent et al. (2000) - [32] & $78 \%$ & $97.3 \%$ & NA & NA \\
\hline
\end{tabular}

\section{Discussion}

The method of 12-lead simultaneous recording of electrocardiographs allows the capturing of the ECG signal of the same cardiac cycle on 12 leads at the same time. This approach can significantly increase the accuracy of all measurements and reduce the variability of ECG measurement [33]. In a multi-lead ECG, the leads refer to imaginary lines between two ECG electrodes. To exploit this information and better model possible beat and lead correlations, we use a third-order tensor structure and then we attempt to extract different feature representations containing complementary information with regard to the dynamics of ECG signals. This fact justifies the superiority of the proposed method against all other state of the art approaches in Table I. While discrete wavelet transform has been widely used in the past for modeling ECG signals, to the best of our knowledge this is the first time that linear dynamical systems, and their projection to Grassmann manifold, are used for the modeling of such data. Linear dynamical systems have shown great ability to model dynamical information in video sequences [34], while recently they have been used for the extension of residual networks, i.e., ResNets, and the improvement of Faster-CNN network's accuracy in object detection applications [35]. The experimental results in Figure 8 show that the combination of LDS descriptors with those extracted from DWT increases significantly the detection rates of individual feature representations presented in Figure 7. 
While both fusion approaches outperform all other state of the art approaches in Table 1, we can notice that the first approach, based on the mapping of both Grassmannian and Euclidean features in a Reproducing Kernel Hilbert Space, provides excellent results in both cases, i.e., detection and localization. In other words, even when the number of classes increases in the case of localization, the discrimination ability of the method remains extremely high, i.e., localization accuracy $100 \%$ for proposed-1 against that of $99.7 \%$ for proposed-2. This is mainly because in the case of VLAD encoding, we mostly keep a statistical information associated with the spatial distribution of descriptors in the geometrical subspaces, while in the case of mapping of feature representations in a Reproducing Kernel Hilbert Space, we are able to better maintain the dynamics and appearance information of the signals encoded in the descriptors. We have to notice that both approaches proposed in the paper are generic and can be easily applied to other application fields, where the fusion of feature representations that belong to different geometrical subspaces and are extracted from either shallow or deep classifiers is required.

\section{Conclusions}

In this paper, we presented a novel methodology for assisting doctors in detection and localization of MI. The main advantage of the proposed approach is that it exploits better the intercorrelations between signals of different ECG leads by extracting feature representations that lie in different geometrical spaces and contain complementary information with regard to the dynamics of signals. More specifically, we initially reshape the multidimensional signal into a third-order tensor structure and subsequently extract feature representations in both Euclidean and Grassmannian spaces. Moreover, two different methods are proposed for the mapping of two different feature representations into a common Hilbert space before the final classification of signals. The first approach is based on the mapping of both Grassmannian and Euclidean features in a Reproducing Kernel Hilbert Space (RKHS), while the second one attempts to apply VLAD encoding directly to Grassmann manifold and then concatenate the two VLAD representations. The experimental results showed that the proposed method improved significantly the performance of the automated computer-based detection and localization of MI. In the future, more data will be collected in order to assess the 
effectiveness of the proposed methodology. Finally, we aim to extend and apply the proposed methodology to other application fields, using different types of signals e.g. EEG or EMG, in order to provide a generalized automated electrodiagnostic tool.

\section{Acknowledgement}

The research leading to these results has received funding from EC under grant agreement no. H2020-690494 “i-PROGNOSIS”.

\section{Conflict of Interest Statement}

We declare that we have no financial and personal relationships with other people or organizations that can inappropriately influence our work, there is no professional or other personal interest of any nature or kind in any product, service and/or company that could be construed as influencing the position presented in, or the review of, the manuscript entitled, "Multi-lead ECG Signal Analysis for Myocardial Infarction Detection and Localization through the Mapping of Grassmannian and Euclidean Features into a Common Hilbert Space".

\section{References}

[1] World Health Organization, Cardiovascular diseases (CVDs). http://www.who.int/news-room/factsheets/detail/cardiovascular-diseases-(cvds)/, (2018) (accessed 14 June 2018).

[2] National Heart, Lung, and Blood Institute, What is a Heart Attack. https://www.nhlbi.nih.gov/health/health-topics/topics/heartattack/, (2018) (accessed 2 July 2018)

[3] L. Sun, Y. Lu, K., Yang, S. Li, ECG analysis using multiple instance learning for myocardial infarction detection, IEEE transactions on biomedical engineering, 59 (12) (2012) 3348-3356. https://doi.org/10.1109/TBME.2012.2213597

[4] E.J.D.S. Luz, W. R. Schwartz, G. Cámara-Chávez, D. Menotti, ECG-based heartbeat classification for arrhythmia detection: A survey. Computer methods and programs in biomedicine, 127 (2016) 144-164. https://doi.org/10.1016/j.cmpb.2015.12.008

[5] U.R. Acharya, H. Fujita, S.L. Oh, Y. Hagiwara, J.H. Tan, M. Adam, Application of deep convolutional neural network for automated detection of myocardial infarction using ECG signals, Information Sciences, 415 (2017) 190-198. https://doi.org/10.1016/j.ins.2017.06.027 
[6] O. Faust, U.R. Acharya, T. Tamura, Formal design methods for reliable computer-aided diagnosis: a review, IEEE reviews in biomedical engineering, 5 (2012) 15-28. https://doi.org/10.1109/RBME.2012.2184750

[7] D. Giri, U.R. Acharya, R.J. Martis, S.V. Sree, T.C. Lim, T.A. VI, J.S. Suri, Automated diagnosis of coronary artery disease affected patients using LDA, PCA, ICA and discrete wavelet transform, Knowledge-Based https://doi.org/10.1109/RBME.2012.2184750

[8] M. Kumar, R.B. Pachori, U.R. Acharya, An efficient automated technique for CAD diagnosis using flexible analytic wavelet transform and entropy features extracted from HRV signals, Expert Systems with Applications, 63 (2016) 165-172. https://doi.org/10.1016/j.eswa.2016.06.038

[9] Q. Zhao, L. Zhang, ECG feature extraction and classification using wavelet transform and support vector machines, in: Proceedings of the IEEE International Conference on Neural Networks and Brain, 2, (2005), pp. 1089-1092. https://doi.org/10.1109/ICNNB.2005.1614807

[10] E.S. Jayachandran, Analysis of myocardial infarction using discrete wavelet transform, Journal of medical systems, 34 (6) (2010) 985-992. https://doi.org/10.1007/s10916-009-9314-5

[11] D. Sadhukhan, S. Pal, M. Mitra, Automated ECG analysis using Fourier harmonic phase, in: Proceedings of the IEEE Region 10 Symposium (TENSYMP), 2017, pp. 1-5. https://doi.org/10.1109/TENCONSpring.2017.8070022

[12] M. Arif, I.A. Malagore, F.A. Afsar, Detection and localization of myocardial infarction using knearest neighbor classifier, Journal of medical systems, 36 (1) (2012) 279-289. https://doi.org/10.1007/s10916-010-9474-3

[13] L.N. Sharma, R.K. Tripathy, S. Dandapat, Multiscale energy and eigenspace approach to detection and localization of myocardial infarction, IEEE transactions on biomedical engineering, 62 (7) (2015) 1827-1837. https://doi.org/10.1109/TBME.2015.2405134

[14] S. Padhy, S. Dandapat, Third-order tensor based analysis of multilead ECG for classification of myocardial infarction, Biomedical Signal Processing and Control, $31 \quad$ (2017) 71-78. https://doi.org/10.1016/j.bspc.2016.07.007

[15] S. Kiranyaz, T. Ince, M. Gabbouj, Real-time patient-specific ECG classification by 1-D convolutional neural networks, IEEE Transactions on Biomedical Engineering, 63 (3) (2016) 664675. https://doi.org/10.1109/TBME.2015.2468589

[16] M. Zubair, J. Kim, C. Yoon, An automated ECG beat classification system using convolutional neural networks, in: Proceedings of the sixth IEEE International Conference on IT Convergence and Security (ICITCS), (2016), pp. 1-5. https://doi.org/10.1109/ICITCS.2016.7740310 
[17] N. Liu, L. Wang, Q. Chang, Y. Xing, X. Zhou, A Simple and Effective Method for Detecting Myocardial Infarction Based on Deep Convolutional Neural Network, Journal of Medical Imaging and Health Informatics, 8 (7) (2018) 1508-1512. https://doi.org/10.1166/jmihi.2018.2463

[18] S. Padhy, L.N. Sharma, S. Dandapat, Multilead ECG data compression using SVD in multiresolution domain, Biomedical signal processing and control, 23 (2016) 10-18. https://doi.org/10.1016/j.bspc.2015.06.012

[19] G. Doretto, A. Chiuso, Y.N. Wu, S. Soatto, Dynamic textures. International Journal of Computer Vision, 51 (2) (2003) 91-109. https://doi.org/10.1023/A:1021669406132

[20] K. Dimitropoulos, P. Barmpoutis, N. Grammalidis, Higher Order Linear Dynamical Systems for Smoke Detection in Video Surveillance Applications, IEEE Trans. Circuits Syst. Video Techn., 27 (5) (2017) 1143-1154. https://doi.org/10.1109/TCSVT.2016.2527340

[21] S.M. Siddiqi, B. Boots, G.J. Gordon, A constraint generation approach to learning stable linear dynamical systems, In Advances in neural information processing systems (2008) 1329-1336.

[22] G. Arfken, Gram-schmidt orthogonalization. Mathematical methods for physicists, 3 (1985) 516520.

[23] H. Jégou, M. Douze, C. Schmid, P. Pérez, Aggregating local descriptors into a compact image representation, in: Proceedings of the IEEE International Conference on Computer Vision and Pattern Recognition (CVPR), (2010), pp. 3304-3311. https://doi.org/10.1109/CVPR.2010.5540039

[24] V. Kantorov, I. Laptev, Efficient feature extraction, encoding and classification for action recognition, in: Proceedings of the IEEE International Conference on Computer Vision and Pattern Recognition (CVPR), (2014), pp. 2593-2600. https://doi.org/10.1109/CVPR.2014.332

[25] K. Dimitropoulos, P. Barmpoutis, A. Kitsikidis, N. Grammalidis, Classification of Multidimensional Time-Evolving Data using Histograms of Grassmannian Points, IEEE Transactions on Circuits and $\begin{array}{llllll}\text { Systems for } \quad \text { Video } & \text { Technology, } & 28 & \text { (4) }\end{array}$ https://doi.org/10.1109/TCSVT.2016.2631719

[26] A.Y. Wright, A.G. Yang, S.S. Sastry, M. Yi, Robust face recognition via sparse representation, IEEE Trans. Pattern Anal. Mach. Intell. $31 \quad$ (2) (2009) 210-222. https://doi.org/10.1109/TPAMI.2008.79

[27] D. Kastaniotis, I. Theodorakopoulos, G. Economou, S. Fotopoulos, Gait based recognition via fusing information from Euclidean and Riemannian manifolds, Pattern Recognition Letters, 84 (2016) 245-251. https://doi.org/10.1016/j.patrec.2016.10.012

[28] H. Karcher, Riemannian center of mass and mollifier smoothing, Communications on Pure and Applied Mathematics, 30 (5) (1977) 509-541. https://doi.org/10.1002/cpa.3160300502 
[29] [dataset] A.L. Goldberger, L.A. Amaral, L. Glass, J.M. Hausdorff, P.C. Ivanov, R.G. Mark, H.E. Stanley, Physiobank, physiotoolkit, and physionet. Circulation, 101(23), e215-e220.

[30] B. Surawicz, T. Knilans, Chou's Electrocardiography in Clinical Practice E-Book: Adult and Pediatric. Elsevier Health Sciences, 2008.

[31] U.R. Acharya, H. Fujita, M. Adam, O.S. Lih, V.K. Sudarshan, T.J. Hong, J.EW. Koh, Y. Hagiwara, C.C. Chua, K.C. Poo, T.R. San, Automated characterization and classification of coronary artery disease and myocardial infarction by decomposition of ECG signals: A comparative study, Information Sciences, 377 (2016) 17-29, https://doi.org/10.1016/j.ins.2016.10.013.

[32] C.D. Nugent, J.A.C. Webb, N.D. Black, Feature and classifier fusion for 12-lead ECG classification, Medical informatics and the Internet in medicine, 25 (3) (2000) 225-235. https://doi.org/10.1080/146392300750019217

[33] B. Chen, W. Guo, B. Li, R.K. Teng, M. Dai, J. Luo, H. Wang, H., A Study of Deep Feature Fusion based Methods for Classifying Multi-lead ECG. (2018) arXiv preprint arXiv:1808.01721.

[34] K. Dimitropoulos, P. Barmpoutis, N. Grammalidis, Spatio-Temporal Flame Modeling and Dynamic Texture Analysis for Automatic Video-Based Fire Detection, IEEE Transactions on Circuits and $\begin{array}{llllll}\text { Systems for } \quad \text { Video } & \text { Technology, } & 25 & \text { (2) }\end{array}$ https://doi.org/10.1109/TCSVT.2014.2339592

[35] A. Dimou, D. Ataloglou, K. Dimitropoulos, F. Alvarez, P. Daras, LDS-Inspired Residual Networks, IEEE Transactions on Circuits and Systems for Video Technology, (2018), https://doi.org/10.1109/TCSVT.2018.2869680

The original publication can be found in:

https://www.sciencedirect.com/science/article/pii/S1746809419301004 